\title{
An Efficient Approximation for Fractional Differential Equations using Operational Matrix by Hermite Polynomials
}

\author{
Hatice Yalman Kosunalp ${ }^{1 *}$, Mustafa Gulsu
}

${ }^{1}$ Bayburt University, Social Sciences Vocational School, Bayburt, Turkey

${ }^{2}$ Mugla Sitki Kocman University, Faculty of Science, Department of Mathematics, Mugla, Turkey

DOI: $10.36347 /$ sipms.2020.v07i05.004

| Received: 16.05.2020 | Accepted: 28.05.2020 | Published: 30.05.2020

*Corresponding author: Hatice Yalman Kosunalp

Abstract

Original Research Article

This paper aims at the derivation of a new operational matrix of fractional integration for Hermite polynomials, in order to solve the linear form of fractional differential equations (FDEs) in the sense of spectral tau method. To do this, we focus explicitly on the conversion of FDEs into a number of algebraic equations to simplify the problem, subject to pre-defined initial conditions. This is achieved by fractional integration through the Riemann-Liouville sense. We then apply to the proposed strategy to figure out the simplified problem. In order to show the performance of the proposed strategy, we present exact and approximated solutions for a number of examples.

Keywords: Hermite, operational matrix, tau method.

Copyright @ 2020: This is an open-access article distributed under the terms of the Creative Commons Attribution license which permits unrestricted use, distribution, and reproduction in any medium for non-commercial use (NonCommercial, or CC-BY-NC) provided the original author and source are credited.

\section{INTRODUCTION}

Fractional differential equations (FDEs) have been gainning a significant attention in various aspects of science and engineering [1-5]. FDEs are utilised to describe the mathematical behavior of typical engineering and applied sciences problems. Therefore, a critical effort is required to be placed on the solutions of FDEs. A number of methods have been proposed to solve FDEs [6-10]. It is well-known that many FDEs do not have exact solutions which drives researchers to exploit the numerical techniques for obtaining approximated solutions. A popular technique, called spectral method, uses traditional polynomials to efficiently obtain approximate solutions. Recently, spectral methods have been shown as an attractive subject with a continuously growing interest for a high volume of real-world problems.

Recently, the derivation of operational matrix for diverse types of polynomials was carried out to address the initial value problems of FDEs. Existing studies attempt to solve either linear or non-linear type of FDEs with operational matrix strategy. The operational matrix for fractional integration has been successfully derived for different types of polynomials.
Examples of these polynomials are Laguerre [11, 12], Chebyshev [13], Legendre [14], Bessel [15], Bernstein [16], Fermat [17] and Boubaker [18] polynomials. In this study, our main motivation is to establish the operational matrix of fractional integration by Hermite polynomials with the sense of Riemann-Liouville. which is believed to solve multi-order FDEs in a costeffective way.

The most significant feature of the proposed idea is to reduce the complexity of FDEs. For this purpose, we first write the FDEs in integral type. This is to construct a set of algebraic equations with the operational matrix developed. Depending on the number of initial conditions, a specific number of algebraic equations are also created. By putting all algebraic equations in a matrix form, the problem is actually reduced to the solution of an algrebraic equation system. In order to test the accuracy and performance of the proposed idea, we solved a number of representative examples. With the results obtained, either exact or approximated solutions are achieved. The details about the method and examples are provided in the following sections. 


\section{Preliminaries}

\section{Definition of Rieman-Liouville Integration}

Rieman-Liouville integration that is represented by $I^{\alpha}$, where $f(x) \in L_{1}[a, b]$ is introduced as [4]

If $\alpha=0$ then

$$
I^{\alpha} f(x)=\frac{1}{\Gamma(\alpha)} \int_{0}^{x}(x-t)^{\alpha-1} f(t) d t \quad \alpha>0, x>0
$$

$$
I^{0} f(x)=f(x)
$$

The following properties are hold by Rieman-Liouville operator: [22]

- $\quad I^{\alpha} I^{\beta}=I^{\beta+\alpha}$ (commutative property)

- $\quad I^{\alpha} I^{\beta}=I^{\beta} I^{\alpha} \quad$ (semi-group property)

- $\quad I^{\alpha} x^{c}=\frac{\Gamma(\alpha+1)}{\Gamma(\alpha+1+c)} x^{\alpha+c}$ (power function)

- $\quad I^{\alpha}\left(c_{1} f_{1}+c_{2} f_{2}\right)=I^{\alpha}\left(c_{1} f_{1}\right)+I^{\alpha}\left(c_{2} f_{2}\right), f(x) \in L_{1}[a, b]$ (linearity)

\section{Method of Operational Matrix}

\section{Hermite Polynomials}

Hermite polynomials are defined on $(-\infty, \infty)$ with this analytical formula: [20]

$$
H_{i}(x)=\sum_{k=0}^{\left\lfloor\frac{i}{2}\right\rfloor} \frac{(-1)^{k} i !(2 x)^{i-2 k}}{k !(i-2 k) !}
$$

where $\left\lfloor\frac{i}{2}\right\rfloor$ denotes the smallest number greatest than $\frac{i}{2}$.

Hermite polynomials are orthogonal since [23]

$$
\int_{-\infty}^{\infty} H_{i}(x) H_{j}(x) w(x) d x=h_{j} \delta_{i j}
$$

where $\delta_{i j}=\left\{\begin{array}{ll}0 & i \neq j \\ 1 & i=j\end{array}\right.$ indicates the function of Kronecker and $h_{j}=2^{j} j ! \sqrt{\pi}$.

\section{Hermite Operational Matrix Method of Solution}

Let Hermite operational matrix is shown as $P^{\alpha}$ with Rieman-Liouville integration and let $\psi(x)$ is the Hermite vector, then it will be

$$
I^{\alpha} \psi(x)=P^{(\alpha)} \psi(x)
$$

Here $P^{(\alpha)}$ is the $(N+1) \times(N+1)$ matrix and matrix elements can be found as below:

$\varphi(n, k)=\sum_{i=0}^{\lfloor n / 2\rfloor} \sum_{r=0}^{\left\lfloor^{k} / 2\right\rfloor} \frac{1}{2^{r} r ! \sqrt{\pi}} \frac{(-1)^{i+r} n ! j ! 2^{n-2 i+k-2 r} \cdot \Gamma\left({ }^{n-2 i+\alpha+k-2 r+1 / 2)}\right.}{2 . i ! r !(k-2 r) ! \Gamma(n-2 i+v+1)}, k=0,1,2 \ldots N$.

Firstly, for finding the elements of $P^{(\alpha)}$ matrix Rieman-Liouville integration of Hermite polynomials is found, it is

$$
I^{\alpha}\left(H_{n}(x)\right)=\sum_{i=0}^{[n / 2]} \frac{(-1)^{i} \Gamma(\mathrm{n}-2 \mathrm{i}+1) 2^{n-2 i+\alpha}\left[(x)^{n-2 i+\alpha}\right]}{(n-2 i) ! i ! \Gamma(\mathrm{n}-2 \mathrm{i}+1+\alpha)}
$$

Then if we think

$$
\left[(x)^{n-2 i+\alpha}\right]=\sum_{k=0}^{N} c_{k} H_{k}(x)
$$

$c_{k}$ can be obtained as below

$$
c_{k}=\frac{1}{2^{k} k ! \sqrt{\pi}} \sum_{r=0}^{\left\lfloor^{k} / 2\right\rfloor} \frac{(-1)^{r} k ! 2^{k-2 r} \Gamma\left(\frac{n-2 i+\alpha+k-2 r+1}{2}\right)}{2 \cdot r !(k-2 r) ! \Gamma(n-2 i+\alpha+1)}
$$

By using (4.3) and (4.4) we obtain

$$
I^{\alpha}\left(H_{n}(x)\right)=\sum_{k=0}^{N} \varphi(n, k) H_{k}(x), \quad n=0,1,2, \ldots, N .
$$


The (4.6) equation is

$$
I^{\alpha}(x) H_{n}=\sum_{k=0}^{N} \sum_{i=0}^{\lfloor n / 2\rfloor} \sum_{r=0}^{\lfloor k / 2\rfloor} \frac{1}{2^{k} k ! \sqrt{\pi}} \frac{(-1)^{i+r} n ! k ! 2^{n-2 i+k-2 r} \cdot \Gamma(n-2 i+v+k-2 r+1)}{2 . i ! r !(k-2 r) ! \Gamma(n-2 i+v+1)} H_{k}(x)
$$

where

$$
\varphi(i, r)=\sum_{i=0}^{\lfloor n / 2\rfloor} \sum_{r=0}^{\lfloor k / 2\rfloor} \frac{1}{2^{j} j ! \sqrt{\pi}} \frac{(-1)^{i+r} n ! k ! 2^{n-2 i+k-2 r} \cdot \Gamma(n-2 i+v+k-2 r+1)}{2 . i ! r !(k-2 r) ! \Gamma(n-2 i+v+1)} .
$$

And the fractional differential equation model we will consider is

$$
D^{\alpha} u(x)=\sum_{J=1}^{k} \gamma_{j} D^{\beta_{J}} u(x)+\gamma_{k+1} u(x)+f(x),
$$

with initial conditions

$$
u^{(i)}(0)=d_{i}
$$

where $\gamma_{j,(j=0,1,2, \ldots, k+1)}$ are real constants and $m-1<\alpha \leq m$, and $0<\beta_{1}<\beta_{2}<\cdots<\beta_{k}<\alpha$ and $f(x)$ is source function. Rieman-Liouville integral of order $\alpha$ is applied to fractional differential equation

$u(x)-\sum_{j=0}^{m-1} u^{(j)}\left(0^{+}\right) \frac{x^{j}}{j !}=\sum_{i=1}^{k} \gamma_{i} I^{\alpha-\beta_{i}}\left[u(x)-\sum_{j=0}^{m_{i}-1} u^{(j)}\left(0^{+}\right) \frac{x^{j}}{j !}\right]+\gamma_{k+1} I^{\alpha} u(x)+I^{\alpha} f(x)$

$u^{(i)}(0)=d_{i}, i=0, \ldots, m-1$

where $m_{i}-1<\beta_{i} \leq m_{i}, m_{i} \in N$. It is obvious that

$$
\begin{gathered}
u(x)=\sum_{i=1}^{k} \gamma_{i} I^{\alpha-\beta_{i}} u(x)+\gamma_{k+1} I^{\alpha} u(x)+g(x), \\
u^{(i)}(0)=d_{i}, i=0, \ldots, m-1
\end{gathered}
$$

where

$$
g(x)=I^{\alpha} f(x)+\sum_{j=0}^{m-1} u^{(j)}\left(0^{+}\right) \frac{x^{j}}{j !}+\sum_{i=1}^{k} \gamma_{i} I^{\alpha-\beta_{i}}\left[u(x)-\sum_{j=0}^{m_{i}-1} u^{(j)}\left(0^{+}\right) \frac{x^{j}}{j !}\right]
$$

The matrix forms of $u_{N}(x)$ and $g(x)$ will be

$$
\begin{aligned}
u_{N}(x) & \cong \sum_{i=0}^{N} c_{i} H_{i}(x)=C^{T} \phi(x) \\
g(x) & \cong \sum_{i=0}^{N} g_{i} H_{i}(x)=G^{T} \phi(x)
\end{aligned}
$$

where the vector $G=\left[\begin{array}{llll}g_{0} & g_{1} & \ldots & g_{N}\end{array}\right]^{T}$ can be calculated whereas $C=\left[\begin{array}{llll}c_{0} & c_{1} & \ldots & c_{N}\end{array}\right]^{T}$ is unkown vector.

We then appply Rieman-Liouville integral of order $\alpha$ and $\left(\alpha-\beta_{j}\right)$ of the approximate solution and we obtain

$$
I^{\alpha} u_{N}(x) \cong C^{T} I^{\alpha} \phi(x) \cong C^{T} \boldsymbol{P}^{(\alpha)} \varphi(x)
$$

and

$$
I^{\alpha-\beta_{j}} u_{N}(x) \cong C^{T} I^{\alpha-\beta_{j}} \phi(x) \cong C^{T} \boldsymbol{P}^{\left(\alpha-\beta_{j}\right)} \varphi(x), j=1,2, \ldots, k
$$

The residual $R_{N}(x)$ will be given as $[14,19]$

with Tau method, by applying (Cite ref)

$$
R_{N}(x)=\left(C^{T}-C^{T} \sum_{i=1}^{k} \gamma_{i} I^{\alpha-\beta_{i}}-\gamma_{k+1} P^{(\alpha)}-G^{T}\right) \phi(x)
$$

$$
\left\langle R_{N}(x), H_{j}(x)\right\rangle=\int_{-\infty}^{\infty} R_{N}(x) . H_{j}(x) w(x) d x=0, j=0,1, \ldots, N-m .
$$

$N-m+1$ linear algebraic equations are generated. From the conditions we have $m$ conditions, by thinking both of them together we will solve $N+1$ equations with Tau method. [24] The coefficients $\left[c_{0}, c_{1}, \ldots, c_{N}\right]$ will be found, by putting them into the equation form [25] such as

$$
u_{N}(x) \cong \sum_{i=0}^{N} c_{i} H_{i}(x)=C^{T} \phi(x)
$$

Then approximate solutions will be found.

\section{Numerical Examples}

Example 1. We first consider the following equation [26]

$$
D^{0.5} u(x)+u(x)=x^{2}+\frac{2 x^{1.5}}{\Gamma(2.5)} u(0)=0,0<x<1 .
$$

where the exact solution is given as

$$
u(x)=x^{2}
$$


Our solution is applied for $\mathrm{N}=2$ and the approximate solution is written as

$$
u_{N}(x)=\sum_{i=0}^{2} c_{i} H_{i}(x)=C^{T} \phi(x) \text { and } g(x)=\sum_{i=0}^{2} g_{i} H_{i}(x)=G^{T} \phi(x)
$$

We also obtain

$$
\mathrm{P}^{0.5}=\left[\begin{array}{ccc}
0.3901 & 0.2885 & 0.0488 \\
0.3847 & 0.3901 & 0.1443 \\
-0.1580 & 0.1923 & 0.2925
\end{array}\right]
$$

The following algebraic equations are extracted, one from the initial condition:

$$
\begin{aligned}
1.3901 c_{0}+0.3847 c_{1}-0.1560 c_{2} & =0.6560 \\
0.2885 c_{0}+1.3901 c_{1}+0.1923 c_{2} & =0.1923 \\
c_{0}-2 c_{2} & =0
\end{aligned}
$$

The solution of this algebraic equations is

$$
\left[\begin{array}{lll}
c_{0} & c_{1} & c_{2}
\end{array}\right]=\left[\begin{array}{lll}
0.5 & 0 & 0.25
\end{array}\right]
$$

Finally, our proposed method achieves the exact solution by:

$$
u_{N}(x)=\left[\begin{array}{lll}
c_{0} & c_{1} & c_{2}
\end{array}\right]\left[\begin{array}{l}
H_{0}(x) \\
H_{1}(x) \\
H_{2}(x)
\end{array}\right]=x^{2}
$$

Example 2. The second example is [12]

$$
D^{5 / 2} u(x)+3 u(x)=3 x^{3}+\frac{6 x^{1 / 2}}{\Gamma(1.5)}, \quad u(0)=0, u^{\prime}(0)=0, u^{\prime \prime}(0)=0
$$

The exact solution is given as

$$
u(x)=x^{3}
$$

We also obtain the following

$$
\mathrm{P}^{2.5}=\left[\begin{array}{cccc}
0.0780 & 0.0962 & 0.0488 & 0.0120 \\
0.0550 & 0.0780 & 0.0481 & 0.0163 \\
-0.0867 & -0.0824 & -0.0195 & 0.0080 \\
-0.2098 & -0.2600 & -0.1237 & -0.0195
\end{array}\right]
$$

The required algebraic equations are found, three of which come from the initial conditions:

$$
\begin{aligned}
1.2340 c_{0}+0.1649 c_{1}-0.2600 c_{2} & -0.6295 c_{3}=0.0449 \\
8 c_{2}=0 & \\
c_{0}-2 c_{2}= & 0 \\
2 c_{1}-12 c_{3}= &
\end{aligned}
$$

Now after applying our technique for $N=3$ we get

$$
\left[\begin{array}{llll}
c_{0} & c_{1} & c_{2} & c_{3}
\end{array}\right]=\left[\begin{array}{llll}
0 & 0.75 & 0 & 0.125
\end{array}\right]
$$

And the approximate solution is the same as exact solution like

$$
u_{N}=u(x)=x^{3}
$$

Example 3. The third example is [27]

$$
D^{2} u(x)+D^{0.75} u(x)+u(x)=x^{3}+6 x+\frac{8.53333 x^{2.25}}{\Gamma(0.25)}, \quad u(0)=0, u^{\prime}(0)=0
$$

The exact solution is given as:

$$
u(x)=x^{3}
$$


We obtain the following fractional integrations for $\mathrm{N}=3$,

$$
\begin{aligned}
\mathrm{P}^{2} & =\left[\begin{array}{cccc}
0.1250 & 0.1410 & 0.0625 & 0.0118 \\
0.0940 & 0.1250 & 0.0705 & 0.0208 \\
-0.1250 & -0.0940 & 0 & 0.0235 \\
-0.3385 & -0.3750 & -0,1410 & 0
\end{array}\right] \\
\mathrm{P}^{1.25} & =\left[\begin{array}{cccc}
0.2345 & 0.2232 & 0.0733 & 0.0047 \\
0.1984 & 0.2345 & 0.1116 & 0.0244 \\
-0.1804 & -0.0496 & 0.0879 & 0.0651 \\
-0.6303 & -0.5411 & -0,0744 & 0.0879
\end{array}\right]
\end{aligned}
$$

The required algebraic equations are composed, two of which come from initial conditions.

$$
\begin{gathered}
1.3595 c_{0}+0.2925 c_{1}-0.3054 c_{2}-0.9688 c_{3}=0.1196 \\
1.3643 c_{0}+1.3595 c_{1}-0.1436 c_{2}-0.9161 c_{3}=0.8754 \\
c_{0}-2 c_{2}=0 \\
2 c_{1}-12 c_{3}=0
\end{gathered}
$$

Solving these algrebraic equations, the coefficients are obtained as

$$
\left[\begin{array}{cccc}
c_{0} & c_{1} & c_{2} & c_{3}
\end{array}\right]=\left[\begin{array}{llll}
0.0209 & 0.7204 & 0.0104 & 0.1201
\end{array}\right]
$$

Then, the approximated solutiin for this example is found as

$$
u_{N}(x)=\left[\begin{array}{llll}
c_{0} & c_{1} & c_{2} & c_{3}
\end{array}\right]\left[\begin{array}{l}
H_{0}(x) \\
H_{1}(x) \\
H_{2}(x) \\
H_{3}(x)
\end{array}\right]=0.96 x^{3}+0.041 x^{2}
$$

We see from the approximated solution that it converges to the exact solution very closely, which is presented in figure 1 below.

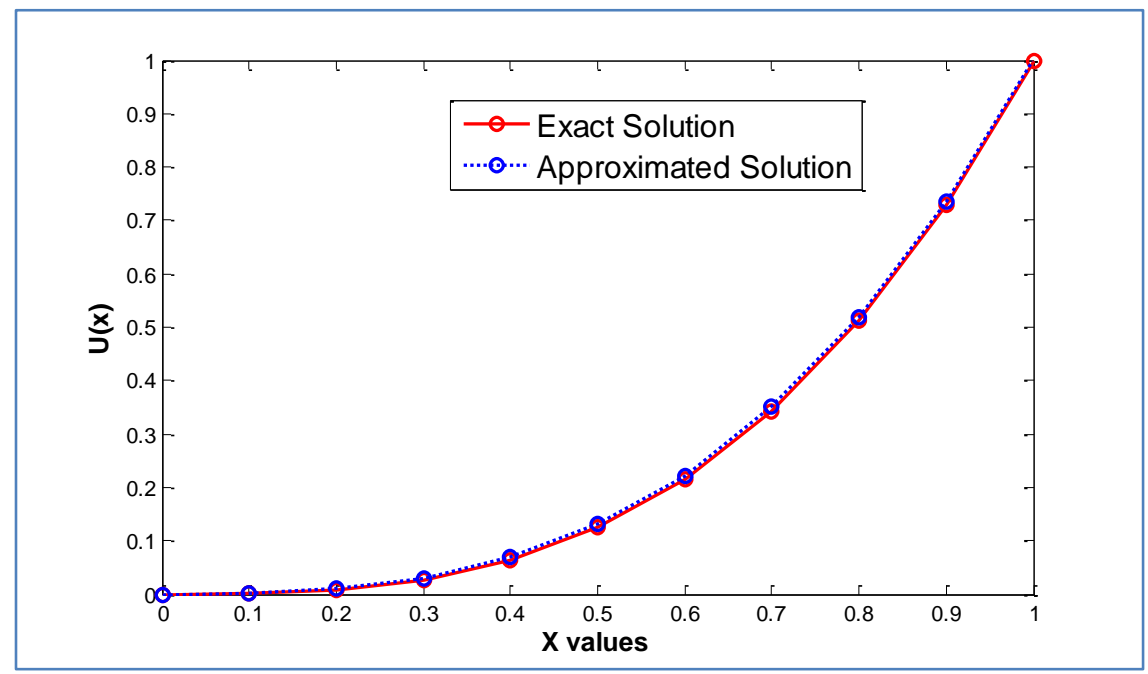

Fig-1: The results of the exact and approximated solutions.

\section{CONCLUSIONS}

This paper presented an explicit derivation of operational matrix of fractional integration through Hermite polynomials. It relies basically on solutions of fractional differential equations with RiemannLiouville. We considered the linear type of fractional differential equations with initial conditions. The basic mechanism is based on the conversion of FDEs into a set of algebraic equations for simplicity. We proved the performance of the proposed mechanism with a number of numerical examples, presenting exact and approximated solutions with high accuracy.

\section{REFERENCES}

1. Diethelm K, Baleanu D, Scalas E. Fractional calculus: Models and numerical methods. World Scientific; 2012.

2. Plonka A. Recent developments in dispersive kinetics. Progress in Reaction Kinetics and Mechanism. 2000 Jul;25(2):109-217.

3. Allegrini P, Buiatti M, Grigolini P, West BJ. Fractional Brownian motion as a nonstationary process: An alternative paradigm for DNA sequences. Physical Review E. 1998 Apr 1;57(4):4558. 
4. Bisquert J. Fractional diffusion in the multipletrapping regime and revision of the equivalence with the continuous-time random walk. Physical review letters. 2003 Jul 3;91(1):010602.

5. Baillie RT. Long memory processes and fractional integration in econometrics. Journal of econometrics. 1996 Jul 1;73(1):5-9.

6. El-Sayed AM, El-Kalla IL, Ziada EA. Analytical and numerical solutions of multi-term nonlinear fractional orders differential equations. Applied Numerical Mathematics. 2010 Aug 1;60(8):788-97.

7. Momani S, Odibat Z. Homotopy perturbation method for nonlinear partial differential equations of fractional order. Physics Letters A. 2007 Jun 11;365(5-6):345-50.

8. Dal F. Application of variational iteration method to fractional hyperbolic partial differential equations. Mathematical Problems in Engineering. 2009;2009.

9. Odibat Z, Momani S, Xu H. A reliable algorithm of homotopy analysis method for solving nonlinear fractional differential equations. Applied Mathematical Modelling. 2010 Mar 1;34(3):593600.

10. Diethelm K, Ford NJ, Freed AD. A predictorcorrector approach for the numerical solution of fractional differential equations. Nonlinear Dynamics. 2002 Jul 1;29(1-4):3-22.

11. Ben-Yu G, Xiao-Yong Z. A new generalized Laguerre spectral approximation and its applications. Journal of Computational and Applied Mathematics. 2005 Sep 15;181(2):342-63.

12. Bhrawy AH, Alghamdi MM, Taha TM. A new modified generalized Laguerre operational matrix of fractional integration for solving fractional differential equations on the half line. Advances in Difference Equations. 2012 Dec 1;2012(1):179.

13. Doha EH, Bhrawy AH, Ezz-Eldien SS. A Chebyshev spectral method based on operational matrix for initial and boundary value problems of fractional order. Computers \& Mathematics with Applications. 2011 Sep 1;62(5):2364-73.

14. Bhrawy AH, Alofi AS. The operational matrix of fractional integration for shifted Chebyshev polynomials. Applied Mathematics Letters. 2013 Jan 1;26(1):25-31.

15. Paraskevopoulos PN, Sklavounos PG, Georgiou GC. The operational matrix of integration for Bessel functions. Journal of the Franklin Institute. 1990 Jan 1;327(2):329-41.
16. Singh AK, Singh VK, Singh OP. The Bernstein operational matrix of integration. Applied Mathematical Sciences. 2009 Feb;3(49):2427-36.

17. Youssri YH. A new operational matrix of Caputo fractional derivatives of Fermat polynomials: an application for solving the Bagley-Torvik equation. Advances in Difference Equations. 2017 Dec $1 ; 2017(1): 73$.

18. Bolandtalat A, Babolian E, Jafari H. Numerical solutions of multi-order fractional differential equations by Boubaker polynomials. Open physics. 2016 Jan 1;14(1):226-30.

19. Belgacem R, Bokhari A, Amir A. Bernoulli Operational Matrix of Fractional Derivative for Solution of Fractional Differential Equations.

20. Poularikas AD. The Handbook of Formulas and Tables for Signal Processing, Hermite Polynomials; 1999.

21. Saadatmandi A. Bernstein operational matrix of fractional derivatives and its applications. Applied Mathematical Modelling. 2014 Feb 15;38(4):136572.

22. Kaçar E, Kaçar Z, Yıldırım H. Integral Inequalities for Rieman-Liouville Frcational Integral of a Function with Respect to Another Function, Iranian Journal of Science and Informatics.2018;13(1):1-13.

23. Zhukovsky K, Dattoli G, Srivastava HM. Orthogonality Properties of Hermite Polynomials and Related Polynomials, Journal of Computational and Applied Mathematics.2005; 182(1): 165-172.

24. Canuto C, Hussaini MY, Quaerteroni TA, Zang TA. Spectral Methods in Fluid Dynamic, PrenticeHall, Englewood Cliffs, NJ;1988.

25. Saadatmandi A, Dehghan M. A new operational matrix for solving fractional-order differential equations. Computers \& mathematics with applications. 2010 Feb 1;59(3):1326-36.

26. Arikoglu A, Ozkol I. Solution of fractional differential equations by using differential transform method. Chaos, Solitons \& Fractals. 2007 Dec 1;34(5):1473-81.

27. Doha EH, Bhrawy AH, Ezz-Eldien SS. Efficient Chebyshev spectral methods for solving multi-term fractional orders differential equations. Applied Mathematical Modelling. 2011 Dec 1;35(12):566272. 